\title{
Urbane Landwirtschaft im Ruhrgebiet
}

\author{
Verdrängung und kreative Resistenz
}

Für Max Weber war Landwirtschaft in der vorindustriellen Stadt noch selbstverständlich. Die meisten Städte „hatten zum mindesten beträchtliche Viehweiden und Waldungen, die ihren Bürgern als solchen zu Gebote standen. Und sehr große Ackerfluren als Zubehör des städtischen Weichbildes sind [...] vorgekommen.“(Weber 1999 [1921]: 67f.) Ein Teil der Bürger*innen war sowohl im Handwerk oder Handel als auch landwirtschaftlich tätig. Daneben gab es „Ackerbürgerstädte“, deren Bürger*innen hauptberuflich Bäuer*innen waren. Hier konnte „eine breite Schicht ansässiger Bürger ihren Bedarf an Nahrungsmitteln eigenwirtschaftlich decken und sogar auch für den Absatz produzieren“" (ebd.).

Seit der Industrialisierung werden jedoch in Stadtforschung und Belletristik Positionen vertreten, nach denen die Landwirtschaft nicht mehr zur Stadt gehöre, obwohl die Statistik der Flächennutzung die große, andauernde Relevanz der urbanen Landwirtschaft für Städte und Ballungsräume in Deutschland durchaus zeigt. Für das Ruhrgebiet, das zu den größten Ballungsräumen in Europa gehört und zugleich einen hohen agrarischen Anteil aufweist, wird im Folgenden der landwirtschaftliche Wandel seit der Industrialisierung skizziert. Er ist von Verdrängungsprozessen, aber auch von der Kreativität geprägt, mit der urbane Landwirt*innen sich bisher behaupten.

\section{Aversionen}

Seit dem Spätmittelalter stehen die Bäuer*innen in der gesellschaftlichen Hierarchie weit unten, auf die die ,besseren' Stände verächtlich herabsehen (Museum 1978; Gerhard 2012). Dieses abwertende Verhältnis bekam mit der Industrialisierung neue Nahrung, als Industrie und Städte den Fortschritt verkörperten, während zum Beispiel Karl Marx und Friedrich Engels den „Idiotismus des Landlebens“ anprangern (Marx/Engels 1982 [1848]: 30). Verbreitet gilt, was Stefan Lessenich schreibt: „Agrargesellschaft [...]: Das klingt nach Tradition und Vormoderne, nach Provinzialität und Rückständigkeit, nach Kuhmist und Bauerntrampel.“ (Lessenich 2018: 82) 
Landleben und Agrargesellschaft stehen somit in einem Gegensatz zur Stadt, die als fortschrittlich gilt. Es erscheint als folgerichtig, wenn Stadtsoziologen wie Hartmut Häußermann und Walter Siebel die Landwirtschaft ausgrenzen und erklären, dass Landwirt*innen gar nicht zur Stadt gehören: „Die Stadt ist Ort der besonderen Lebensform derer, die nicht in der Landwirtschaft tätig sind“ (Häußermann/Siebel 1987: 7), da nur der „Städter ist, wer nicht mehr tagtäglich mit einer unkultivierten Natur ums eigene Überleben kämpfen muss" (Siebel 2004: 21).

Diese Einschätzung, dass die urbane Landwirtschaft nicht zur Stadt gehöre, schlägt sich im Ruhrgebiet durchaus in der realen Stadtentwicklung seit der Industrialisierung nieder. So wollte die Stadt Bochum bereits 1870 mit der Privatisierung ihrer Gemeinschaftsweide (,Vöhde') auch ihre angebliche bäuerliche Rückständigkeit überwinden, die mit dem Viehtreiben durch Bochumer Straßen und dem Spottnamen ,Kaubaukum' verbunden war.[1] Der Stadtpark, der auf der früheren Vöhde angelegt wurde, galt ,als leuchtendes Zeichen des Fortschritts“" (Häpke 2012: 141). Ähnlich sieht es keine hundert Jahre später auch der Schriftsteller und Städter Heinrich Böll, als er fordert, die Agrarflächen im Ruhrgebiet in Parks umzuwandeln:

„Es gibt ihn noch, unverändert hübsch und echt: den vestischen Bauernhof, es gibt die Kuh vor dem Förderturm, weidende Schafe, säende, mähende Bauern, Garben und Ackerkrume, aber diese hübschen Bilder [...] sehen aus, als wären sie bestellt, um fotografiert zu werden; [...] sentimental und verlogen [...]. Wenn man schon den Fortschritt proklamiert, sollte man an ihn glauben: die Häuser in die Höhe bauen - und aus den Äckern Parks machen; [...] wahrscheinlich wären die riesigen Weiden als Parks nützlicher." (Böll/Chargesheimer 1958: 24 f.)

Inzwischen ist es der ökologische Fortschritt, dem die urbane Landwirtschaft weichen muss. Ein erstes Beispiel hierfür brachte die Internationale Bauausstellung Emscher Park, die von 1989 bis 1999 im Ruhrgebiet stattfand. Sie entwickelte auf Landwirtschaftsflächen ein Baugebiet (Backumer Tal) mit der Begründung, dass damit „die gesamtökologische Situation im Vergleich zur bisherigen landwirtschaftlichen Nutzung verbessert"werde (IBA 1993: 225).

Trotz dieser Ausgrenzungen und Verdrängungen ist die urbane Landwirtschaft immer noch ein wichtiges Element der städtischen Realität.

\section{2. Ökonomisch unbedeutend - aber flächenwirksam}

Ökonomisch ist die Landwirtschaft unbedeutend: Land-, Forstwirtschaft und Fischerei tragen 2018 nur zu 0,7 Prozent zum Bruttoinlandsprodukt in Deutschland bei und stellen nur 1,4 Prozent der Erwerbstätigen (Statistisches Bundesamt 2019: 11; Arbeitskreis 2019: 260).

Der Eindruck der Bedeutungslosigkeit ändert sich, wenn die Flächen betrachtet werden. Bundesweit wird die Hälfte des Bodens landwirtschaftlich genutzt (UBA 2019). In den zehn einwohnerstärksten Städten der BRD[2] (Brinkwirth et al. 2019) wird fast ein Viertel ihrer Stadtflächen von 1.500 Agrarbetrieben genutzt.[3] Im Ruhrgebiet leben in 53 Städten und Gemeinden 5,1 Millionen Menschen auf einer Gesamtfläche von 4.440 Quadratkilometern. Davon entfallen sogar 37 Prozent auf die 
Landwirtschaft. Rund 3.400 Betriebe sind aktiv (IT.NRW 2019: 33111-o3iz, 41141-01i). Damit ist die urbane Landwirtschaft das wichtigste Element der ,Grünen Infrastruktur‘ in den Städten.[4]

Insofern ist die Situation in Deutschland anders als in Nordamerika, wo urban agriculture flächenmäßig oftmals eher kleine Projekte bezeichnet (Sbicca o. D.; Lovell 2010; Tornaghi 2014)[5], die die Dimensionen der bundesdeutschen städtischen Landwirtschaft nicht erreichen. Dagegen umfasst die ,Urbane Landwirtschaft' in Deutschland professionelle landwirtschaftliche und gartenbauliche Betriebe in und am Rande von städtischen Ballungsräumen. Ihr Ziel ist die kontinuierliche Produktion von Lebensmitteln oder Dienstleistungen und ihre Vermarktung, um das Einkommen der landwirtschaftlichen Familien zu erwirtschaften (Pölling/ Born 2015: 377). Dabei gelten besondere städtische Bedingungen: So nützt der urbanen Landwirtschaft die Nähe zu den Verbraucher*innen, während sie unter Umweltbelastungen, hohen Pachtflächenanteilen und kurzen Pachtlaufzeiten leidet. Die größten Herausforderungen sind jedoch eine extreme Flächenknappheit und die ständigen Flächenverluste zugunsten von Siedlungstätigkeit und Naturschutz. Wie geht die urbane Landwirtschaft im Ruhrgebiet damit um?

\section{Moderne Stadtentwicklung dank Markenteilung}

Die Flächenverluste haben - wenig überraschend - mit der Industrialisierung begonnen und wurden damals - kaum bekannt - mit einem Instrument der Agrarreformen, den Marken- und Gemeinheitsteilungen durchgesetzt. Zur Erläuterung ein kurzer Rückblick:

Bis in das 19. Jahrhundert hinein war die Hälfte aller landwirtschaftlichen Flächen in Westfalen kein Privat-, sondern Gemeinschaftseigentum, so auch die Agrarflächen der Bürger*innen im städtischen Umfeld. Diese ,Allmenden“ waren gemeinschaftliche Viehweiden, verbunden mit Holzgewinnung oder temporärem Ackerbau, und hießen in Westfalen ,Mark', ,Gemeinheit' oder ,Vöhde' (Häpke 2012: 40-46).

Fürsten wie Friedrich II. von Preußen und der Bischof von Münster wollten seit dem 17. Jahrhundert diese Gemeinschaftsflächen parzellieren und privatisieren, weil sie sich Wirtschaftswachstum und freiwerdende Flächen für neue Untertanen versprachen. Obwohl die Markenteilungen zunächst erfolgreich waren, kehrten viele Bauern im Alltag zur gemeinschaftlichen Nutzung von Gehölzen und Weiden zurück (Häpke 2012: 43 ff.). Mit der Industrialisierung wurden die Städte die treibenden Kräfte. Historisch bedingt waren sie Mitglieder in den Markgenossenschaften und hatten Anteile an den Gemeinschaftsflächen. Sie waren an den Teilungen und Privatisierungen interessiert, um frei verfügbare Grundstücke für den Städtebau zu bekommen. So legte die Stadt Bochum auf ihrem Flächenanteil einen kommunalen Stadtpark und drumherum ein Villenviertel an (Häpke 2012: 155 f.). Ähnlich wurden auf den städtischen Anteilen der parzellierten Marken die Dortmunder Nordstadt, die Oberhausener Innenstadt und weitere Siedlungen gebaut, deren Entstehung noch nicht aufgearbeitet ist (Häpke 2012: $47 \mathrm{f}$.). 


\section{Urbane Pacht-Landwirtschaft}

Mit den Markenteilungen[6] wurde das Privateigentum am landwirtschaftlichen Boden und sein Warencharakter durchgesetzt. Dabei wurden den Bäuer*innen ebenfalls private Anteile an den Gemeinschaftsweiden zugeteilt, die sie wo möglich in Ackerland umgewandelt haben. Aufgrund produktionstechnischer Fortschritte (bei Düngung und Fruchtfolgen) konnten sie auch steigende Flächenerträge erzielen. Die städtische Latrine und Thomasschlacke, ein Abfallprodukt der Stahlerzeugung, waren günstig verfügbare Dünger. Trotz Flächenverlusten ist die Zahl der Bauernhöfe in der zweiten Hälfte des 19. Jahrhunderts daher gewachsen (Häpke 2012: 49 f.).

Mit der industriellen Entwicklung nahmen jedoch die Umweltschäden zu. Der Rauch der Industrie schlug sich auf Obst, Gemüse und Weiden nieder. Der untertägige Steinkohlenbergbau verursachte Geländeabsenkungen und störte die Vorflut, betroffene Felder vernässten (Häpke 2012: 51). Da etliche Bäuer*innen noch durch die Ablösung von Feudallasten im Zuge der Preußischen Agrarreformen verschuldet waren, haben viele ihre nunmehr privaten Parzellen verkauft, wenn sie von Umweltschäden betroffen oder bedroht waren. Erfolgreiche Käufer waren Bergbau und Industrie, die so Schadensersatz vermieden und viele Flächen zurückverpachtet haben. Seit damals arbeitet die urbane Landwirtschaft im Ruhrgebiet auf umfangreichen Pachtflächen, die oft kurzfristig kündbar sind (Häpke 2012: 49-52). Im Durchschnitt sind heute rund 6o Prozent der Agrarflächen im Ruhrgebiet gepachtet, in den Großstädten Dortmund, Duisburg und Oberhausen sogar 70 bis 80 Prozent (IT.NRW 2019: 41141-04i).

\section{Abmelkwirtschaft und partielle Selbstversorgung der Arbeiter*innen}

Der industrielle Arbeitskräftebedarf im Ruhrgebiet war so groß, dass sogar Menschen aus Polen angeworben wurden. Ihnen wurde um 1900 viel versprochen:

„umgeben von Feldern, Wiesen und Wäldern, den Vorbedingungen guter Luft, liegt [...] eine reizende, ganz neu erbaute Kolonie der Zeche Viktor [...]. Zu jeder Wohnung gehört ein sehr guter, hoher und trockener Keller, so daß die eingelagerten Früchte, Kartoffeln etc. dort sehr gut erhalten werden. Ferner gehört dazu ein geräumiger Stall, wo sich jeder sein Schwein, seine Ziege oder seine Hühner halten kann. So braucht der Arbeiter nicht jedes Pfund Fleisch oder sein Liter Milch zu kaufen. Endlich gehört zu jeder Wohnung auch ein Garten [...]. So kann sich jeder sein Gemüse, sein Kumpst und seine Kartoffeln, die er für den Sommer braucht, selbst ziehen. Wer noch mehr Land braucht, kann es in der Nähe von Bauern billig pachten“ (zitiert nach: Steinborn 1991: 16).

Das Grün war ein zentrales Argument, um Bergarbeiter anzuwerben. Gärten waren für viele Menschen unverzichtbar für ihre Lebensmittelversorgung, so dass versprochene Parzellen sehr attraktiv waren. In diesen ,Kolonien“ lebte nur ein Teil der Arbeiterschaft. Andere wohnten in Mietwohnungen, zur 
Untermiete oder gar als ,Schlafgänger‘. Wer von ihnen einen Garten nutzen wollte und neben der ,Maloche' noch bewältigen konnte, konnte sich der Schrebergartenbewegung anschließen oder ,Grabeland ' auf Industrie- oder Eisenbahngelände pachten (Häpke 2012: 50 ff.).

Auch die Bäuer*innen unterstützten die Arbeiterselbstversorgung, indem sie einzelne Felder mit Kartoffeln bestellten, parzellierten und als ,Feldgärten ‘ vermieteten. Unterbrochen durch die Hunger- und Krisenjahre während und nach dem Ersten Weltkrieg, setzte damals die Kundenorientierung der urbanen Landwirtschaft ein. Sie konzentrierte sich auf Marktlücken, erzeugte Getreide für Bäckereien und Kornbrennereien, vor allem aber Milch und Rindfleisch. Milch war als Fett- und Eiweißquelle unverzichtbar, ohne Kühlschränke und nicht pasteurisiert aber kaum lagerfähig und musste immer frisch angeboten werden. Da Luftverschmutzung und Flächenknappheit die Kälberaufzucht im Revier verhinderten, bildete sich die ,Abmelkwirtschaft heraus: Bäuer*innen im Ruhrgebiet kauften im Umland Kühe, die gerade gekalbt hatten, holten sie in ihre Ställe, molken sie und versorgten so die Menschen mit Frischmilch. Wenn die Milchleistung nachließ, wurden die Kühe geschlachtet (Häpke 2012: 50).

Seit den 1930er Jahren ging die Abmelkwirtschaft zurück, nicht nur weil Pasteurisierung und Kühlgeräte sich verbreiteten, sondern auch weil das NS-Regime im Zuge von Autarkiepolitik und Kriegsvorbereitung eine totale Kontrolle der Milchwirtschaft anstrebte und den Ab-Hof-Verkauf von Milch sowie das Buttern auf den Höfen verboten und die dafür erforderlichen Handzentrifugen beschlagnahmt hat. Nach und nach wurde die lokal erzeugte Milch durch Molkereimilch und - billigere - Importe verdrängt (Fink-Keßler 2012: 116, 162 ff.).

\section{Nachkriegszeit und „Wirtschaftswunder“}

Zunächst machten der Zweite Weltkrieg und seine Zerstörungen die Selbstversorgung so wichtig, dass auch in öffentlichen Grünanlagen Kartoffeln angebaut wurden. Das ,Wirtschaftswunder' brachte die Wende: Die Bevölkerung verlor das Interesse an der Selbstversorgung, in Kleingärten wurden Gemüsebeete in Zierrasen umgewandelt, während die professionelle urbane Landwirtschaft mit der Schweine- und Geflügelhaltung neue Marktlücken nutzen konnte. Trotzdem blieb ihre Viehdichte unter der im ländlichen Raum, um Streit mit der Nachbarschaft über tierische Gerüche zu vermeiden. Massentierhaltung und eine das Grundwasser gefährdende Gülledüngung sind im Ruhrgebiet, anders als zum Beispiel im ländlichen Münsterland kein Problem. Die wieder zunehmenden Flächenverluste im Ruhrgebiet - von 1950 bis 1974 ein Viertel insgesamt, absolut 40.000 Hektar ( $=5$ Hektar pro Tag) (Statistisches Landesamt Nordrhein-Westfalen 1950, 1974) - erforderten neue Einkommensquellen, denn als Pächter*innen hatten die meisten urbanen Landwirt*innen von Baulandverkäufen keinen Nutzen. Immerhin konnten sie mehr Brotgetreide statt Futterhafer anbauen, weil mit der Motorisierung Pferde als Zugtiere unnötig wurden. Zugleich begannen die urbanen Landwirt*innen mit eigenen Verkaufswagen durch die umliegenden Wohngebiete zu fahren, um Eier und Kartoffeln zu verkaufen. 


\section{7. Ökologische Konflikte und urbane Landwirtschaft}

Gegen Ende der 1960er Jahre gerieten die BRD, vor allem das Ruhrgebiet in eine Wirtschaftskrise. Das ,Zechensterben“ wurde unübersehbar. Wenige Jahre später kam die Umweltbewegung hinzu, doch das wachsende Umweltbewusstsein hatte widersprüchliche Folgen. Da die (,konventionelle') Landwirtschaft nach dem Zweiten Weltkrieg zunehmend Agrochemikalien einsetzte, erstarkte als Gegenbewegung der ökologische Landbau. Im Ruhrgebiet lag der Kern des alternativen Landbaus in den 1980ern im Umfeld des Kernkraftwerks in Hamm; die dortigen Biobäuer*innen waren wichtige Kräfte der Anti-AKW-Bewegung (Häpke 2012: 72 ff.). Ansonsten aber hat der ökologische Landbau im Ruhrgebiet kaum Chancen, weil die mehrjährige Umstellungszeit für die kurzfristig kündbaren Pachtlandwirte ökonomisch zu riskant ist. Daher betreiben hier nur 2,7 Prozent der Betriebe auf 2,o Prozent der Flächen ökologischen Landbau, während weiter südlich im ländlichen Raum zehn bis 15 Prozent der Betriebe biologisch wirtschaften (IT.NRW 2019: 41141-O2i).

Zugleich ist die Naturschutzszene stärker geworden. Seitdem wird die Landwirtschaft von zwei Seiten, in die Zange' genommen. Während die Siedlungs- und Verkehrsflächen zu Lasten der Landwirtschaft ständig wachsen, müssen die damit verbundenen Eingriffe in Natur und Landschaft ,ausgeglichen' werden, insbesondere durch Pflanzung von Hecken, Obstbäumen oder durch Aufforstungen. Auch dies geschieht häufig auf Flächen der Landwirtschaft. Andererseits liegen im Ruhrgebiet umfangreiche Industrie- und Gewerbebrachen, die nicht wieder genutzt werden, weil sie industrielle Altlasten enthalten, deren Beseitigung niemand bezahlen will. Daher werden Altlasten nur gesichert und unter Grünanlagen getarnt (Häpke 2012: 239 ff.). Für Wohnen und Gewerbe werden hingegen Agrarflächen überplant. Ein wichtiger Grund sind die Bodenwertzuwächse: Während 2017 im Ruhrgebiet für Bauland zwischen 175 und 450 Euro je Quadratmeter gezahlt werden, kosten landwirtschaftliche Flächen nur zwischen 2 und 6 Euro je Quadratmeter. Trotzdem ist die Verdrängung der Landwirtschaft nicht allein finanziell zu erklären, da Forstflächen mit 1,60 Euro je Quadratmeter noch billiger sind (GMB 2018: 43, 54, 56). Doch die Forstwirtschaft hat erreicht, dass jede Abholzung mindestens flächengleich durch Aufforstung ersetzt werden muss (Häpke 2012: 104 ff.). Davon kann die Landwirtschaft nur träumen.

\section{Diversifizierung und Kundenorientierung}

Nachdem der Agrarsektor im Ruhrgebiet (rechtsrheinisch) von 1979 bis 2001 erneut 16 Prozent seiner Flächen verloren hat (3 Hektar täglich) (LDS 1979, 2001), wurden von 2001 bis 2015 weitere 10.00o Hektar anderen Nutzungen zugeführt (2 Hektar pro Tag). Davon wurde eine Hälfte zu Siedlungs- und Verkehrsflächen, während die andere Hälfte aufgeforstet wurde (IT.NRW 2019: 33111-04iz). Diese Flächenverluste bewirken, dass jährlich fast 2 Prozent der Bauernhöfe aufgeben, während andere versuchen, ihre Wertschöpfung zu erhöhen.

Eine verstärkte Tierhaltung oder die Erzeugung von Biogas sind in der Stadt wegen der Emissionen nicht möglich. Stattdessen werden in der Stadt 
landwirtschaftliche Gebäude, wie Scheunen, für Festivitäten vermietet; Kindergeburtstage werden organisiert, aber auch Schulklassen und Kindergartengruppen können landwirtschaftliche Betriebe als außerschulischen ,Lernort Bauernhof besuchen, um die Entfremdung der Städter*innen von ihren Lebensmitteln zu überwinden (CoProGrün 2018a).

Die urbanen Landwirt*innen verstärken ihre Kundenorientierung, erweitern ihre Direktvermarktung und eröffnen eigene Hofläden und Hofcafés. Neue Trends sind Verkaufsautomaten und Milchtankstellen, die rund um die Uhr in Betrieb sind. Während früher vor allem Zuwandererfamilien nach der Ernte liegengebliebene Kartoffeln einsammeln durften, werden inzwischen Erdbeeren, Bohnen oder Blumen eigens angebaut, damit sie von den Kund*innen selbst gepflückt werden (Häpke 2012: 72 ff.; RVR/LWK 2014; Pölling/ Born 2015). Hinzu kommen Mietgärten, die von Landwirt*innen streifenweise mit Gemüse und Kräutern bestellt und parzelliert an Städter*innen zur weiteren Pflege und Ernte vermietet werden. Start-ups wie Ackerhelden bieten ergänzende Beratungen an (CoProGrün 2017). Eine neue Form der Zusammenarbeit ist die ,Solidarische Landwirtschaft'. Hier kaufen die Verbraucher*innen keine einzelnen Produkte, sondern übernehmen einen festen Anteil an den betrieblichen Kosten, zahlen einen regelmäßigen Mitgliedsbeitrag und bekommen einen festen Anteil an der Ernte, egal wie diese ausfällt (CoProGrün 2018b).

\section{Zusammenfassung}

Nachdem für Max Weber, einen der Begründer der Soziologie, zu Beginn des 20. Jahrhunderts die städtische Landwirtschaft noch selbstverständlich war, wird sie inzwischen von mehreren Seiten in Frage gestellt: aus literarischer und soziologischer Sicht, von der Stadtentwicklung wie vom Naturschutz. Praktisch unterliegt die urbane Landwirtschaft seit der Industrialisierung im Ruhrgebiet einem Verdrängungsprozess, indem ständig Agrarflächen für Siedlung, Verkehr, aber auch Naturschutz in Anspruch genommen werden. Während dabei viele Betriebe aufgeben, versuchen andere mit Kreativität, diesem Druck zu widerstehen und Anerkennung zu gewinnen. Entscheidend ist dabei die Kooperation der Landwirt*innen mit den Stadtbewohner*innen: die Erzeugung von Mangelprodukten wie der Milch in der Weimarer Zeit und davor, die Fahrt der Bäuer*innen mit Verkaufswagen durch Wohngebiete, die Kund*innen, die Hofläden besuchen, verschiedene Formen von Selbsterntefeldern bis zu der bisher erst seltenen Solidarischen Landwirtschaft. Die Umweltbildung und die Vermittlung von Naturerlebnissen, die insbesondere auf urbanen Bauernhöfen für die städtische Bevölkerung angeboten werden, wirken der Naturentfremdung entgegen. Auf diese Weise hat sich im Ruhrgebiet eine spezifisch urbane Landwirtschaft konstituiert, die sich durch eine große Multifunktionalität auszeichnet. 


\section{Endnoten}

[1] Kaubaukum bedeutet, Kuh-Bochum‘ und soll ausdrücken, dass Bochum keine aufstrebende Industriestadt, sondern ein ,Kuh-Dorf geblieben sei.

[2] Berlin, Hamburg, München, Köln, Frankfurt am Main, Stuttgart, Düsseldorf, Dortmund, Leipzig, Essen.

[3] Eigene Berechnungen nach Daten der statistischen Landesämter.

[4] Auf ihre mit der akuten Klimakrise zunehmende Bedeutung kann hier nicht eingegangen werden.

[5] Für diese Literaturhinweise danke ich Justin Kadi.

[6] ,Markenteilung‘ ist die Aufteilung einer größeren gemeinschaftlich genutzten Fläche in kleinere Parzellen, die an die einzelnen Mitglieder der bisherigen Nutzergemeinschaft zur privaten weiteren Nutzung verteilt werden.

\section{Autor innen}

Ulrich Häpke ist Raumplaner mit den Schwerpunkten Freiraumschutz, urbane Landwirtschaft, Naturschutz durch Naturnutzung, Ruhrgebiet und Nachhaltigkeit.

u.haepke@web.de

\section{Literatur}

Böll, Heinrich / Chargesheimer, Karl-Heinz (1958): Im Ruhrgebiet. Frankfurt am Main: Büchergilde Gutenberg.

Brinkwirth, Jacqueline / Wirth, Sophie von / Berndt, Gero (2019): Die zehn größten Städte Deutschlands im Ranking. In: Handelsblatt, 31.5.2019. www.handelsblatt.com/politik/ deutschland/ranking-2019-die-zehn-groessten-staedte-deutschlands/24407466.html (letzter Zugriff am 28.9.2019).

CoProGrün (2017): Dokumentation des Workshops „Landwirtschaftliche Co-Produktion und Vermarktung“ am 07.11.2017. www.coprogruen.de/files/coprogruen_-_dokumentation_ workshop_produktion___vermarktung.pdf (letzter Zugriff am 27.3.2019).

CoProGrün (2018a): Der Grünzug als Bildungs- und Sozialort. www.coprogruen.de/files/ coprogruen_-_dokumentation_workshop_bildung_und_soziales.pdf (letzter Zugriff am 27.3.2019).

CoProGrün (2018b): Projektbörse CoProGrün. www.coprogruen.de/veranstaltungen/ projektboerse-maerz-2018.html (letzter Zugriff am 27.3.2019).

Fink-Keßler, Andrea (2012): Milch. München: oekom.

Gerhard, Gesine (2012): Das Bild des Bauern in der modernen Industriegesellschaft. In: Daniela Münkel / Frank Uekötter (Hg.): Das Bild des Bauern. Göttingen: Vandenhoeck \& Ruprecht, 111-130.

GMB 2018 - Der Obere Gutachterausschuss für Grundstückswerte im Land NRW (Hg.) (2018): Grundstücksmarktbericht 2018. Düsseldorf.

Häpke, Ulrich (2012): Freiraumverluste und Freiraumschutz im Ruhrgebiet. Common-Property-Institutionen als Lösungsansatz? Dortmunder Beiträge zur Raumplanung. Blaue Reihe 139. Dortmund: IRPUD.

Häußermann, Hartmut / Siebel, Walter (1987): Neue Urbanität. Frankfurt am Main: Suhrkamp.

IBA - Internationale Bauausstellung Emscher Park (Hg.) (1993): Katalog zum Stand der Projekte. Gelsenkirchen.

Lessenich, Stefan (2018): Neben uns die Sintflut. München: Piper.

Lovell, Sarah Taylor (2010): Multifunctional Urban Agriculture for Sustainable Land Use Planning in the United States. In: Sustainability 2010/2, 2499-2522.

Marx, Karl / Engels, Friedrich (1982 [1848]): Manifest der Kommunistischen Partei. In: Karl Marx / Friedrich Engels: Ausgewählte Schriften in zwei Bänden. Band I. Berlin: Dietz. 
Museum für Deutsche Volkskunde Berlin (Hg.) (1978): Das Bild vom Bauern. Vorstellungen und Wirklichkeit vom 16. Jahrhundert bis zur Gegenwart. Berlin.

Pölling, Bernd / Born, Rolf (2015): Urbane Landwirtschaft in der Metropole Ruhr. In: Natur und Landschaft 9o/o8, 376-382.

RVR/LWK - Regionalverband Ruhr / Landwirtschaftskammer NRW (Hg.) (2014): Bauernhoferlebnisse in der Metropole Ruhr, Heft 1: Emscher Landschaftspark. Essen.

Sbicca, Joshua (o. D.): Urban Agriculture, Revalorization, and Green Gentrification In Denver, Colorado. www.researchgate.net/publication/331337777_Urban_ Agriculture_Revalorization_and_Green_Gentrification_in_Denver_Colorado/ link/5c796ac9a6fdcc4715a6cc85/download (letzter Zugriff am 28.9.2019).

Siebel, Walter (2004): Einleitung. In: Walter Siebel (Hg.): Die europäische Stadt. Frankfurt am Main: Suhrkamp, 11-50.

Steinborn, Vera (Hg.) (1991): Arbeitergärten im Ruhrgebiet. Kleine Reihe 5. Dortmund: Westfälisches Industriemuseum.

Tornaghi, Chiara (2014): Critical geography of urban agriculture. In: Progress in Human Geography 38/4, 551-567.

Weber, Max (1999[1921]): Wirtschaft und Gesellschaft. Teilband 5: Die Stadt (hg. von Wilfried Nippel). Tübingen: J.C.B.Mohr (Paul Siebeck).

\section{Statistiken}

Arbeitskreis „Erwerbstätigenrechnung des Bundes und der Länder” (Hg.) (2019): Erwerbstätige in den kreisfreien Städten und Landkreisen der BRD 1991 bis 2017. Wiesbaden.

IT.NRW (2019): Landesdatenbank. www.landesdatenbank.nrw.de/ldbnrw/online (letzter Zugriff am 28.9.2019), eigene Berechnungen.

LDS - Landesamt für Datenverarbeitung und Statistik NRW (versch. Jg.): Bodenflächen in NRW. Düsseldorf.

Statistisches Bundesamt (2019): Bruttoinlandsprodukt 2018 für Deutschland. Begleitmaterial zur Pressekonferenz am 15. Januar 2019 in Berlin. Wiesbaden.

Statistisches Landesamt Nordrhein-Westfalen (versch. Jg.): Statistisches Jahrbuch Nordrhein-Westfalen. Düsseldorf.

UBA - Umweltbundesamt (2019): Struktur der Flächennutzung. www. umweltbundesamt.de/daten/flaeche-boden-land-oekosysteme/flaeche/struktur-derflaechennutzung\#textpart-1 (letzter Zugriff am 21.9.2019). 